\title{
EVIDENCE FOR OVERDOMINANT SELECTION MAINTAINING X-LINKED FITNESS VARIATION IN DROSOPHILA MELANOGASTER
}

\author{
Tim Connallon ${ }^{1}$ And L. LaCEy KNOWLes ${ }^{2}$ \\ Department of Ecology and Evolutionary Biology and Museum of Zoology, 2019 Natural Science Building, 830 North University, \\ University of Michigan, Ann Arbor, Michigan 48109-1048 \\ ${ }^{1}$ E-mail: tconnal@umich.edu \\ 2E-mail: knowlesl@umich.edu
}

\begin{abstract}
The role of balancing selection in maintaining genetic variation for fitness is largely unresolved. This reflects the inherent difficulty in distinguishing between models of recurrent mutation versus selection, which produce similar patterns of inbreeding depression, as well as the limitations of testing such hypotheses when fitness variation is averaged across the genome. Signatures of X-linked overdominant selection are less likely to be obscured by mutational variation because X-linked mutations are rapidly eliminated by purifying selection in males. Although models maintaining genetic variation for fitness are not necessarily mutually exclusive, a series of predictions for identifying X-linked overdominant selection can be used to separate its contribution from other underlying processes. We consider the role of overdominant selection in maintaining fitness variation in a sample of $12 \mathrm{X}$ chromosomes from a population of Drosophila melanogaster. Substantial variation was observed for male reproductive success and female fecundity, with heterozygous-X genotypes exhibiting the greatest degree of variance, a finding that agrees well with predictions of the overdominance model. The importance of X-linked overdominant selection is discussed along with models of recurrent mutation and sexually antagonistic selection.
\end{abstract}

Key words.-Balancing selection, fitness variation, overdominant selection, sex-by-genotype interaction, sexual antagonism, $\mathrm{X}$ chromosome.

Received February 28, 2006. Accepted May 17, 2006.

Elucidating mechanisms that maintain naturally segregating genetic variation, particularly variation affecting fitness, is one of the fundamental problems in evolutionary genetics (Lewontin 1974). Large amounts of genetic variation in fitness related traits, as well as for total fitness, have been reported by many studies (e.g., Tracey and Ayala 1974; Gardner et al. 2005), raising the question of whether selection, rather than recurrent mutation by itself, maintains much of the observed fitness variation (Charlesworth and Charlesworth 1999; Charlesworth and Hughes 1999; Saccheri et al. 2005; but see Zhang and Hill 2005). Yet, direct evidence of selectively maintained variation has rarely been demonstrated.

When fitness variation is measured across large portions of the genome, the extent of nonadaptive (i.e., mutational) and adaptive fitness variation is difficult to evaluate. Moreover, most studies have focused on autosomal variation (i.e., at diploid loci), in which relatively high mutational loads may obscure signatures of selection for a minority of loci. The X chromosome can facilitate analysis of selection's role in maintaining fitness variation. Mutational noise is expected to be lower on the $\mathrm{X}$ because deleterious mutations can be efficiently purged through selection in males (Crozier 1976; Eanes et al. 1985; but see Wilton and Sved 1979). Furthermore, theory of $\mathrm{X}$ chromosome evolution can be used to generate predictions (outlined below) for identifying the signature of overdominant selection, a process likely to maintain polymorphism.

Variance among hemizygous-, homozygous-, and heterozygous-X genotypes.-Recurrent deleterious mutation and overdominant selection are expected to generate distinct patterns of fitness variation when they act upon X-linked loci. Recurrent mutation is expected to inflate fitness variance among males (dosage compensation doubling expression of the single $\mathrm{X}$ is assumed) and homozygous females relative to variance among outbred females (hereafter "heterozygous" females, in which individuals inherit unique $X$ chromosomes by descent) (James 1973; our analysis below). This difference in variance arises because extreme phenotypes are rare within samples of heterozygous individuals, but common among homozygous (or hemizygous) individuals.

$\mathrm{X}$-linked overdominant selection is expected to inflate fitness variance among heterozygous females relative to males and homozygous females (see Charlesworth and Hughes 1999). Overdominance maintains X-linked polymorphism when alternative alleles for a locus have similar hemizygous fitness effects - the heterozygous benefit to females must not be overpowered by directional selection for the most favorable allele in males (Pamilo 1979; Hedrick and Parker 1997). Fitness variation among heterozygous (i.e., outbred) females can therefore be substantial (e.g., some individuals at locus $A$ will be homozygous - $A_{1} A_{1} / A_{2} A_{2}$ - and some heterozygous- $A_{1} A_{2}$ ), even when homozygous individuals exhibit little to no fitness variance (e.g. $A_{1} A_{1}$ individuals are about as fit as $A_{2} A_{2}$ ).

Recurrent mutation is expected to increase relative fitness variance among homo- and hemizygous individuals, whereas $\mathrm{X}$-linked overdominant selection will increase variance among heterozygous individuals. The ratio of fitness variance between males or homozygous females versus heterozygous females can be used to infer the relative impact of these processes on X-linked polymorphism.

Mean dominance of alleles influencing female fitness.When segregating genetic variation is partially dominant (i.e., rare variants influence fitness in heterozygous condition), fitness of hetero- and homozygous individuals is expected to be correlated. This expectation underlies methods of estimating the average dominance coefficient, $h$, for mutations 
TABLE 1. Models maintaining X-linked fitness variation and the corresponding genotypic fitness for females and males with alleles $A_{1}$ and $A_{2} \cdot{ }^{1}$

\begin{tabular}{|c|c|c|c|c|c|}
\hline & \multicolumn{3}{|c|}{ Female genotypes } & \multicolumn{2}{|c|}{ Male genotypes } \\
\hline & $A_{1} A_{1}$ & $A_{1} A_{2}$ & $A_{2} A_{2}$ & $A_{1}$ & $A_{2}$ \\
\hline Mutation-selection balance & $1-s$ & $1-s h$ & 1 & $1-t$ & 1 \\
\hline Overdominant selection in females & $1-s$ & 1 & $1-t$ & $1-u$ & $1-v$ \\
\hline Opposing selection (sexual antagonism) & $1-s$ & $1-s h$ & 1 & 1 & $1-t$ \\
\hline
\end{tabular}

${ }^{1}$ Selection coefficients are denoted by variables $s, t, u$, and $v$. Allelic dominance is reflected by $h$. For all variables $(n), 0 \leq n \leq 1$.

affecting fitness (Mukai and Yamaguchi 1974; Eanes et al. 1985; Charlesworth and Charlesworth 1999). A strong positive association reflects additive genetic variation $(h>0)$; nonadditive variation $(h=0)$ decreases the association. The average dominance coefficient is expected to be greater than zero for variation that is maintained by mutation-selection balance, an effect of mildly deleterious alleles that are partially expressed in heterozygotes. Alternatively, when variation is maintained by overdominant selection, estimated dominance is expected to be zero (Charlesworth and Charlesworth 1999).

In this study, we examine variation in female fecundity and male reproductive success, composite traits that are closely associated with fitness. We measure variation among 12 D. melanogaster $\mathrm{X}$ chromosomes in heterozygous, homozygous, and hemizygous (i.e., male) condition, and contrast observations with predictions derived from overdominance and mutation-selection balance models. A third model—sexually antagonistic selection-is also considered in light of the observations (Table 1 describes the three models). The analyses reveal that a proportion of X-linked fitness variation might be maintained by overdominant selection. Additional research using the $\mathrm{X}$ chromosome approach outlined here will be required to assess the generality of these results.

\section{Materials And Methods}

\section{Model and Predictions}

To predict patterns of fitness variance among experimental groups of heterozygous and homozygous individuals, we analyzed two simple genetic models (single- and two-locus) under variable conditions of dominance and allele frequency. For each locus considered, two allele types $\left(A_{1}\right.$ at frequency $p ; A_{2}$ at frequency $q$ ) segregate within the population. Individual effects of each locus can be summed to determine an individual's fitness (as is typically assumed in the quantitative genetics literature; Falconer and Mackay 1996; Charlesworth and Hughes 1999). Heterozygous populations are those in which each individual inherits a unique $\mathrm{X}$ chromosome by descent; genotype frequencies per X-linked locus conform to Hardy-Weinberg expectations. Homozygous populations are completely inbred at X-linked loci (inbreeding coefficient, $F=1$ ) and proportions of each homozygous genotype are proportional to allele frequencies within the population $\left(A_{1} A_{1} / A_{2} A_{2}=p / q\right)$. Because males are effectively homozygous for the $\mathrm{X}$ chromosome (expression of the $\mathrm{X}$ that each carries is doubled by dosage compensation, as occurs in Drosophila species), they are genetically equivalent to the homozygous group. Linkage equilibrium between loci is assumed in all cases.

We first analyzed the effects of dominance coefficients and allele frequencies at a single locus. Consideration of multiple unlinked loci with identical dominance coefficients and at similar frequencies leads to identical results. We then considered a more realistic scenario in which allele frequencies, dominance coefficients, and selection coefficients vary between loci. Results are shown for a two locus model, in which dominance and selection coefficients negatively covary, as is predicted by the theory of mutation-selection balance (i.e., frequencies of mutant alleles are proportional to $\mu / s h$; Hartl and Clark 1997; Crow 1993).

\section{Fly Stocks and Crosses}

Isofemale lines of D. melanogaster were collected in State College, Pennsylvania (by Andrew Clark, 1998; kindly provided by Doris Bachtrog). All other stocks were obtained from the Bloomington Stock Center, Bloomington, Indiana. All flies were reared at $25^{\circ} \mathrm{C}$ on standard cornmeal-yeastsucrose-agar medium.

Twelve "experimental' $\mathrm{X}$ chromosomes (hereafter $X_{i}$ ) were isolated from 12 isofemale lines by crossing males with multiple compound-X $(C(1) D X, y, f)$ females. Males with a target $X_{i}$ were then crossed to females heterozygous for the FM6 balancer chromosome, which eliminates crossing over on the $\mathrm{X}$ chromosome during female gametogenesis. Heterozygous $\mathrm{F}_{1}$ females for the $X_{i}$ and balancer chromosomes were then crossed to: (1) males carrying the same target $X_{i}$ to produce females completely homozygous for the $\mathrm{X}$ chromosome $\left(X_{i} X_{i}\right)$, and experimental males $\left(X_{i} / \mathrm{Y}\right)$, or (2) males carrying a "standard" $X$ chromosome $\left(X_{s}\right.$; also extracted by crossing with $C(1) D X, y, f)$ to produce females with two different $\mathrm{X}$ chromosomes $\left(X_{i} X_{s}\right.$; hereafter referred to as " heterozygous"'), and experimental males $\left(X_{i} / Y\right)$ (Fig. 1). This type of crossing technique is common (Lewontin 1974; Eanes et al. 1985). The paired design here uses the same set of experimental $\mathrm{X}$ chromosomes in heterozygous and homozygous, condition. Therefore associations between $\mathrm{X}$ chromosome and autosomal variation (Fig. 1) should similarly affect fitness in heterozygous, homozygous, and male individuals. Nevertheless, if partially inbred autosomes contribute to inbreeding depression, the fitness variance of homozygous individuals is expected to be inflated (as autosomes carry a substantial recessive mutational load; Charlesworth and Charlesworth 1999; our analysis below). This will make conclusions favoring overdominant selection conservative.

In some previous studies (e.g., Gibson et al. 2002), each 

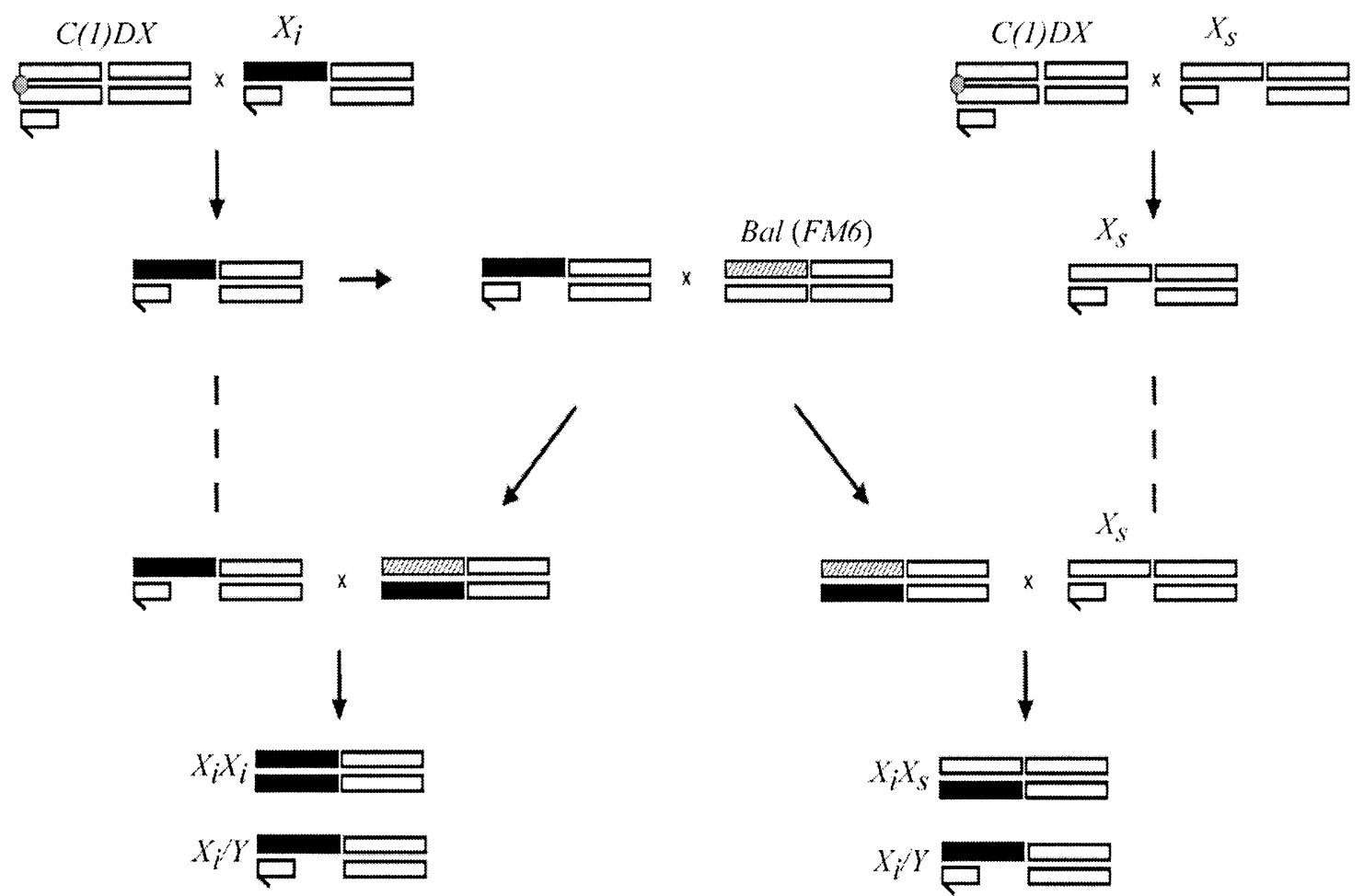

FIG. 1. Crosses used to generate experimental males, homozygous, and heterozygous females. Sex chromosomes and an autosome are shown. Black chromosomes represent experimental $\mathrm{X}$ chromosomes $\left(X_{i}\right)$, shaded chromosomes represent the balancer chromosomes $(F M 6)$, and standard $\mathrm{X}$ chromosomes $\left(X_{s}\right)$ are labeled.

experimental chromosome is tested in combination with many chromosomes and the mean fitness of the set of genotypes is used to estimate heterozygous fitness. This design potentially underestimates fitness variance in the heterozygous group. For example, consider an X-linked locus subject to overdominant selection, in which alternative alleles are each at high frequency (and with similar homozygous fitnesses). Each experimental heterozygote (with two unique $\mathrm{Xs}$ ) has a $\sim 50 \%$ probability of being heterozygous at that locus; $\sim 50 \%$ will be homozygous. Crossing each experimental X to an array of chromosomes and calculating fitness per $\mathrm{X}$ as the average of these will underestimate fitness variation between heterozygous genotypes. Our crossing scheme (Fig. 1) is expected to better estimate fitness variation between individuals in an outbred population, which is a key variable for identifying overdominance.

\section{Fitness Assays}

Previous studies of $\mathrm{X}$-linked variation in D. melanogaster indicate that most fitness variation is expressed during the adult stage (Eanes et al. 1985; Gibson et al. 2002). We therefore focus our attention on adult-expressed variation. Because many characters are likely to reflect fitness in the adult stage, we decided to measure composite traits that incorporate many characteristics influencing male and female reproductive success. It is important to note that although total fitness is not measured, we refer to "fitness" variation throughout for purposes of brevity.

Female fitness._-Adult reproductive success in female Drosophila is predicted to be a function of each female's survival (e.g., Holland and Rice 1999), ability to allocate resources to egg production (Bateman 1948), and ability to find a suitable mate (Partridge 1980; Zeh and Zeh 1996). Our female fitness assay incorporates the latter two important variables.

Two day old homozygous and heterozygous $\left(X_{i} X_{i}\right.$ and $\left.X_{i} X_{s}\right)$ virgin females (10-20 individuals at a time) were mass mated for two days to equal numbers of males taken from an outbred base stock of Pennsylvanian D. melanogaster. Females were then isolated, placed in a fresh food vial, and transferred every third day (for a total of nine days) to avoid larval crowding. Total adult offspring emergence was used as the female fitness estimate. This measure controls for potential biases in offspring provisioning (i.e., egg size) between experimental genotypes. Because there is little X-linked variation affecting egg-to-adult viability (e.g., Eanes et al 1985; Gibson et al. 2002), measuring the number of offspring that a female is able to produce bypasses the egg counting problem and reasonably estimates female fecundity. Fitness was measured for a total of 4662 females, with an average of 187 individuals scored for each of the 25 genotypes (12 X chromosomes in homozygous and heterozygous state; the standard $\mathrm{X}$ in homozygous state).

Male fitness. - In species without male parental investment (e.g., Drosophila spp.) an adult male's fitness is primarily determined by his ability to access and successfully fertilize female eggs (Bateman 1948; Trivers 1972), a function of preand postmating male-male competition and female choice. The male fitness assay incorporates multiple traits that are subject to sexual selection and important in determining male fitness. These include pre- and postcopulatory male-male and male-female interactions. 
Replicates of 10 four-day-old virgin males were placed in competition vials with twenty white eyed $\left(w^{-} / Y\right)$ males and twenty $w^{-} / w^{-}$females. The ratio of experimental to competitor males as well as the overall sex ratio (male biased; $3: 2$ ) was skewed to accentuate multiple aspects of male reproductive success, including sperm competitive ability. White eyed $\left(w^{-} / w^{-}\right)$females were individually placed in vials after $24 \mathrm{~h}$, and permitted to lay eggs for the next three days. The ratio of red-eyed to white-eyed daughters was used to estimate the paternal contribution of experimental compared to competitor males. The average ratio per $\mathrm{X}$ chromosome was taken as a measure of male fitness for that genotype. This type of procedure is common (e.g., Chippindale et al. 2001). Paternity ratios were scored for 2991 white-eyed females, which is equivalent to an average of 125 males per genotype. Mixed broods were common, indicating that sperm competition differences between genotypes are captured in the fitness estimates.

Statistical analyses. - The female fitness estimate for the most fit genotype was set to 1 . All other genotypes were scaled against it. Similarly, the $\mathrm{X}$ associated with the highest fitness in males was set to 1 , and each less successful $X$ scaled against it. Tests for fitness variance between genotypes and within experimental groups (i.e., within males, and homozygous and heterozygous females) were performed using analysis of variances (ANOVA). Linear regression was used to test for relationships between variables.

To test whether the magnitude of fitness variance differed between $\mathrm{X}$ chromosomes in hemizygous, homozygous, and heterozygous, condition, the data was first partitioned so that the fitness of each genotype was scaled against the most fit genotype within the group (e.g., for homozygous females, the best genotype was set to 1 and relative fitness of the other homozygous genotypes were scaled relative to the best). These data were then $r$-transformed, as described in O'Brien (1981). The transformed values for a group have a mean equal to the variance of the original group. Variance differences between groups can therefore be assessed by way of ANOVA. This method is powerful and robust to violations of normality. $T$-tests are two tailed except when otherwise noted. In cases where analyses are one-tailed, means are predicted to deviate in particular directions (e.g., the null hypothesis: $\mu_{1} \leq \mu_{2}$; Sokal and Rohlf 1995). All statistical analyses were conducted with JMP (vers. 4.0, SAS Institute).

To identify potential outliers that might affect the results, quantile box plot analyses (outliers exceed 1.5 interquartile ranges from the median) were performed. A single but obvious outlier, $X_{5}$, was identified (shown in Fig. 2); reported results are without the outlier unless otherwise stated. Removal of this chromosome from our analysis decreases the correlation between males and females, and between homozygous and heterozygous females, suggesting that this chromosome contains one or more highly deleterious, non sex-limited mutations of (roughly) additive expression. Because the aim of this study is to elucidate features specific to the $\mathrm{X}$ chromosome that may lead to the maintenance of fitness variation, and non sex-limited strongly deleterious mutations are unsuitable sources for maintained variation on the $\mathrm{X}$ chromosome, the removal of the outlier is appropriate.

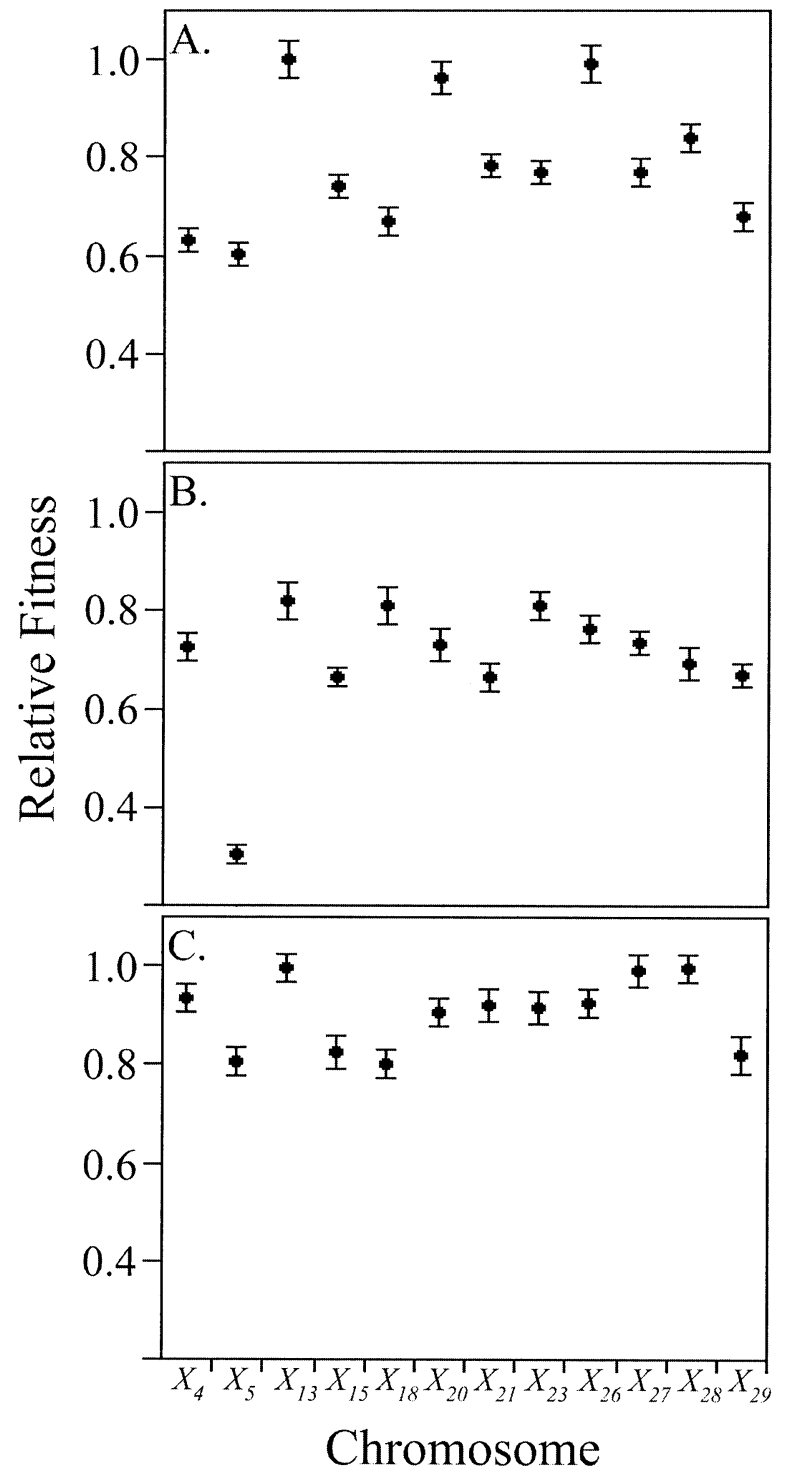

FIG. 2. Distribution of experimental $\mathrm{X}$ chromosome effects on male reproductive success and female fecundity for (A) heterozygous females, (B) homozygous females, and (C) males. Means and standard errors are shown for each chromosomal genotype.

Nevertheless, results are presented with and without the outlier.

\section{RESUlts AND Discussion}

Models that maintain genetic variation for fitness are not necessarily mutually exclusive. When many loci are analyzed concurrently, different processes are probably operating at different loci. Nevertheless, constraints associated with analyzing variation across large segments of the genome (e.g., $\mathrm{X}$ chromosomes) can be overcome when individual processes maintaining polymorphism are relatively important and stand out from the background. Below, we contrast patterns in our data with theoretical predictions of X-linked mutation-selection balance and overdominant selection. We also consider a third model of sexual antagonism, which is likely to influence sex chromosome evolution (Table 1; Rice 1984; Gibson 
TABLE 2. Variance ratios between experimental groups.

\begin{tabular}{llcc}
\hline \hline Time period & \multicolumn{1}{c}{ Comparison } & Ratio & $P^{2}$ \\
\hline Cumulative & homo- vs. heterozygotes & 0.316 & 0.021 \\
& males vs. heterozygotes & 0.296 & 0.019 \\
& homozygotes vs. males & 1.067 & 0.876 \\
First & homo- vs. heterozygotes & 0.308 & 0.030 \\
Second & homo- vs. heterozygotes & 0.911 & 0.424 \\
Third & homo- vs. heterozygotes & 2.506 & 0.961 \\
\hline
\end{tabular}

${ }^{1}$ Time periods are broken up into three day intervals (see methods for details).

${ }^{2} P$-values represent the probability that homozygous or male variance $>$ heterozygous variance; one-tailed (see methods).

${ }^{3} P$-value represents the probability that variance does not differ between groups; two-tailed.

et al. 2002). Our results suggest that overdominant selection has a nontrivial role in maintaining X-linked fitness variation, although this conclusion is given with some caveats and suggestions for future research.

\section{Variance among Hemizygous-, Homozygous-, and Heterozygous-X Genotypes}

Experimental results. - Statistically significant genotypic variance for fitness is evident for homozygous females $\left(X_{5}\right.$ removed: $F_{10,2065}=3.519, P=0.0001 ; X_{5}$ included $F_{11,2275}$ $=21.808, P<0.0001)$, males $\left(X_{5}\right.$ removed: $F_{10,2632}=4.450$, $P<0.0001 ; X_{5}$ included $\left.F_{11,2966}=5.029, P<0.0001\right)$, and heterozygous females $\left(X_{5}\right.$ removed: $F_{10,2017}=16.724, P<$ $0.0001 ; X_{5}$ included $\left.F_{11,2201}=18.974, P<0.0001\right)$ (Fig. 2). Cumulative variance estimates are higher among heterozygous than among homozygous female and male genotypes (hereafter $V_{X_{i} X_{s}}, V_{X_{i} X_{i}}$, and $V_{\text {male }}$, respectively). The latter two groups have approximately equal variance (Table 2 ). The hypothesis that variance in homo- and hemizygous genotypes is higher than variance in heterozygous genotypes can be rejected for the pooled dataset. However, this pattern did not hold when variance estimates were partitioned into three day intervals, where $V_{X_{i} X_{i}}$ is substantially greater than $V_{X_{i} X_{s}}$ during the last interval. This is due to a sharp increase in homozygous variance over time $\left(F_{2,30}=7.03, P=0.0031\right)$; heterozygous variance did not change between intervals $\left(F_{2,30}\right.$ $=1.012, P=0.3756$ ).

Model predictions._Like James (1973), we found that homozygous and hemizygous individuals exhibit twice the variance of X-linked heterozygous individuals, when fitness variation is additive ( $h=0.5$; Table 3$)$. In addition, variance among homozygous and hemizygous genotypes was also greater than $V_{X_{i} X_{s}}$ under most conditions of dominance and allele frequency variation. The only exceptions occur when recessive (or partially recessive) alleles are at high frequency within the population (Table 3 ). When selection intensity and
TABLE 3. Predicted X-linked phenotypic variance ratios for hemizygous (or homozygous) versus heterozygous (i.e., "outbred") populations at a single, polymorphic locus. ${ }^{1,2}$

\begin{tabular}{llllll}
\hline \hline & \multicolumn{5}{c}{ Allele frequencies $(p / q)$} \\
\cline { 2 - 6 }$h^{3}$ & $0.9 / 0.1$ & $0.8 / 0.2$ & $0.7 / 0.3$ & $0.6 / 0.4$ & $0.5 / 0.5$ \\
\hline 0 & 0.58 & 0.69 & 0.84 & 1.04 & 1.33 \\
0.1 & 0.71 & 0.83 & 0.99 & 1.21 & 1.52 \\
0.2 & 0.89 & 1.02 & 1.18 & 1.40 & 1.69 \\
0.3 & 1.13 & 1.26 & 1.42 & 1.61 & 1.85 \\
0.4 & 1.48 & 1.58 & 1.69 & 1.82 & 1.96 \\
0.5 & 2 & 2 & 2 & 2 & 2 \\
0.6 & 2.81 & 2.54 & 2.31 & 2.12 & 1.96 \\
0.7 & 4.07 & 3.18 & 2.59 & 2.17 & 1.85 \\
0.8 & 5.97 & 3.81 & 2.74 & 2.11 & 1.69 \\
0.9 & 8.17 & 4.21 & 2.74 & 1.97 & 1.52 \\
1 & 9.09 & 4.17 & 2.56 & 1.79 & 1.33 \\
\hline
\end{tabular}

${ }^{1}$ Variance is across different homozygous genotypes; the heterozygous population is assumed to be under Hardy-Weinberg equilibrium.

${ }^{2}$ Analytical results can be extended to multiple loci when dominance coefficients are held constant.

3 The dominance coefficient, $h$, refers to the more abundant allele.

dominance coefficients are permitted to vary for alleles at multiple loci, the conditions in which $V_{X_{i} X_{s}}$ exceeds $V_{X_{\mathrm{i}} X_{i}}$ are reduced (Table 4). The least restrictive scenario where $V_{X_{i} X_{i}}$ $\leq V_{X_{i} X_{s}}$-i.e., $h=0, p=0.62$ - is considered in more detail below.

For an X-linked locus, the equilibrium frequency $(p)$ of recessive female-limited mutations is equal to $V(3 u / 2 s)$, where $u$ is the mutation rate and $s$ is the selection coefficient against deleterious alleles (Crozier 1976). When $p>0.62$, $u / s$ must be greater than 0.128 , a value that is orders of magnitude higher than any realistic estimate (e.g., assuming a high mutation rate of $10^{-5}$ per locus, $s$ cannot exceed 7.8 $\left.\times 10^{-5}\right)$. Thus, a mutation-selection balance model is compatible with the fitness variance data (see Table 2) only when the average selection coefficient is of the same order of magnitude as the mutation rate (the modified exact equation (9.13) from Hedrick 1985 leads to the same conclusions). When non sex-limited mutations are considered (a more realistic scenario, because males also exhibit substantial fitness variation; Fig. 2; Table 2), the potential contribution of deleterious recessives to observed fitness variance becomes more unlikely, as conditions necessary for $p>0.62$ become absurdly restrictive.

Predictions of the sexual antagonism model also poorly match the experimental results. Rice (1984) modeled the equilibrium frequency (for females and males, respectively) of an $\mathrm{X}$-linked male-beneficial sexually antagonistic allele, which, when completely recessive $(h=0)$, can be expressed as

TABLE 4. Predicted X-linked phenotypic variance ratios for hemizygous (or homozygous) versus heterozygous (i.e., "outbred") populations when dominance and selection coefficients covary. ${ }^{1}$

\begin{tabular}{llllllllll}
\hline \hline$q^{2}$ & 0.1 & 0.2 & 0.3 & 0.4 & 0.5 & 0.6 & 0.7 & 0.8 & 0.9 \\
$V_{\text {hemi }}: V_{\text {hetero }}$ & 7.62 & 4.12 & 2.74 & 1.91 & 1.55 & 1.24 & 1.03 & 0.87 & 0.74 \\
\hline
\end{tabular}

${ }^{1}$ Analytical results are for two loci, $a$ and $b, h_{a}=0.1, h_{b}=0.2$, and $h_{a} s_{a}=h_{b} s_{b}$, as predicted under mutation-selection balance models (Hartl and Clark 1997), where $h$ represents dominance and $s$ represents the strength of selection.

$2 q$ represents the frequency of mutant alleles. 
TABle 5. Homo- and heterozygote adult female fitness and inbreeding depression estimates for experimental X chromosomes; $95 \%$ confidence intervals are shown.

\begin{tabular}{|c|c|c|c|c|c|c|c|c|}
\hline \multirow[b]{2}{*}{ Mean fitness } & \multicolumn{8}{|c|}{ Time period ${ }^{1}$} \\
\hline & First & $P^{2}$ & Second & $P$ & Third & $P$ & Cumulative & $P$ \\
\hline Homozygotes & $0.79 \pm 0.03$ & $>0.1$ & $0.68 \pm 0.09$ & $>0.1$ & $0.54 \pm 0.10$ & 0.03 & $0.74 \pm 0.04$ & 0.07 \\
\hline Heterozygotes & $0.83 \pm 0.06$ & & $0.72 \pm 0.10$ & & $0.67 \pm 0.08$ & & $0.80 \pm 0.08$ & \\
\hline
\end{tabular}

${ }^{1}$ Time periods are broken up into three day intervals (see Methods for details).

${ }^{2}$ Probability that fitness in heterozygotes $\leq$ homozygotes; one-tailed (see Methods).

${ }^{3}$ As defined by Greenberg and Crow (1960); $L=\ln (A)-\ln (B)$, where $A$ is the fitness of heterozygotes and $B$ is the fitness of homozygotes.

$$
\begin{aligned}
& p_{f}=\frac{s}{2 t(s+1)} \\
& p_{m}=\frac{s(s+1)}{s^{2}+2 t(s+1)}
\end{aligned}
$$

where $s$ is the benefit to males and $t$ the cost to females. Male-beneficial alleles only exceed $p=0.62$ when $s / t>1.25$ (weak selection; $s=0.01$ ) and $s / t>1.45$ (strong selection; $s=0.2$ ). However, $V_{\text {male }}$ is greater than $V_{X_{i} X_{s}}$ and $V_{X_{i} X_{i}}$ whenever $s$ is greater than $t$. Our data, interpreted under the sexual antagonism model, present a conflicting scenario. $V_{X_{i} X_{i}}$ is less than $V_{X_{i} X_{s}}$ whenever $p$ is large (i.e., $>0.62$ ), which requires that $s>t$. Yet, when $s>t$, male variance is expected to be greater than female variance (e.g., $V_{\text {male }} \sim 2 V_{X_{i} X_{s}} \sim 2 V_{X_{i} X_{i}}$ under strong selection, $s / t=1.45)$. The sexual antagonism hypothesis cannot account for large $V_{X_{i} X_{S}}$ relative to small and similar $V_{\text {male }}$ and $V_{X_{i} X_{i}}$ values.

An overdominance model can easily account for observed values of $V_{\text {male }}, V_{X_{i} X_{s}}$, and $V_{X_{i} X_{i}}$. When X-linked, overdominant selected loci are not expressed in males, allele frequencies equilibrate at $p=s /(s+t)$, where $s$ and $t$ refer to fitness declines in the two homozygous genotypes (Fisher 1922; Table 1). For a single locus at Hardy-Weinberg equilibrium, the relative variance of homozygous versus heterozygous genotypes depends on the value of $s$ relative to $t\left(V_{X_{i} X_{i}}<\right.$ $V_{X_{i} X_{s}}$ when $s / t<2.6 ; V_{X_{i} X_{s}}: V_{X_{i} X_{i}}$ approaches infinity as $s / t$ approaches one). For a non sex-limited X-linked locus, where

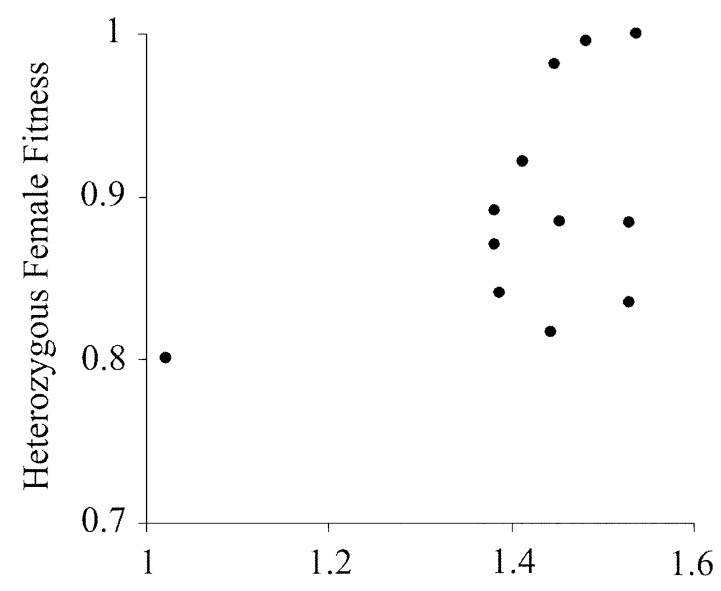

Sum of Homozygous Female Fitnesses

FIG. 3. The relationship between heterozygous female fitness $\left(X_{i} X_{s}\right)$ and the sum of corresponding homozygous fitnesses $\left(X_{i} X_{i}+\right.$ $X_{s} X_{s}$ ). The outlier $X_{5}$ is shown. alleles have identical homozygous fitness effects in females and males ( $s=u, t=v$; Table 1$)$, a stable polymorphism results when $s<3 t-2 t^{2}$ (Pamilo 1979) - that is, when $s$ and $t$ are similar in magnitude $(s / t<2.6$ under strong selection, $s=0.5$; $s / t<3$ under weak selection, $s=0.001)$. In other words, the conditions favoring overdominant polymorphism at $\mathrm{X}$-linked loci tend to increase $V_{X_{i} X_{S}}$ relative to $V_{X_{i} X_{i}}$ and $V_{\text {male. }}$

It should be noted that the possibility that $V_{X_{i} X_{i}}$ is greater than $V_{X_{i} X_{S}}$ during later time periods cannot be rejected (Table $2)$. The ratio of $V_{X_{i} X_{i}}: V_{X_{i} X_{s}}$ increases over time due to an increase in homozygous variance; heterozygous variance does not change. Similarly, the fitness cost associated with X chromosome homozygosity also increases over time (Table 5; 1st vs. 3rd interval, two-tailed $P=0.053$ ). Such patterns are expected under the mutation accumulation theory of senescence, which predicts that deleterious mutations will accumulate more readily when they act late in life-a reflection of weak purifying selection (Medawar 1952; Hughes and Charlesworth 1994; but see Promislow et al. 1996). The increase in homozygous variance over time might therefore reflect an abundance of mutations with late acting deleterious effects. It also indicates that the X chromosome methodology for detecting the signature of overdominant selection might only be feasible during relatively early life history stages.

\section{Allelic Dominance}

The slope of the regression line between heterozygous fitness and the sum of corresponding homozygous fitnesses $\left(X_{i} X_{i}+X_{s} X_{s}\right)$, an estimate of mean dominance of mutant alleles (Mukai and Yamaguchi 1974), is not significantly different from zero $\left(X_{5}\right.$ removed: slope $=0.62 \pm 1.44, R^{2}=$ $0.082, P=0.39 ; X_{5}$ included: slope $=0.21 \pm 0.57, R^{2}=$ $0.16, P=0.19$ ) (Fig. 3), though obviously this analysis lacks precision because of the large standard errors. Nevertheless, homozygous fitness is a poor predictor of heterozygous fitness, suggesting that nonadditive variation and/or completely recessive alleles $(h=0)$ primarily influence female fitness variation.

This finding is compatible with mutation-selection balance and sexual antagonism models when contributing alleles act recessively $(h=0)$. Furthermore, this is the sole expectation of the overdominance model (Charlesworth and Charlesworth 1999; Charlesworth and Hughes 1999). Previous studies with Drosophila species indicate that a high proportion of standing variation for fitness is additively expressed, with mean dominance estimates ranging from 0.15 to 0.40 (reviewed in Sim- 


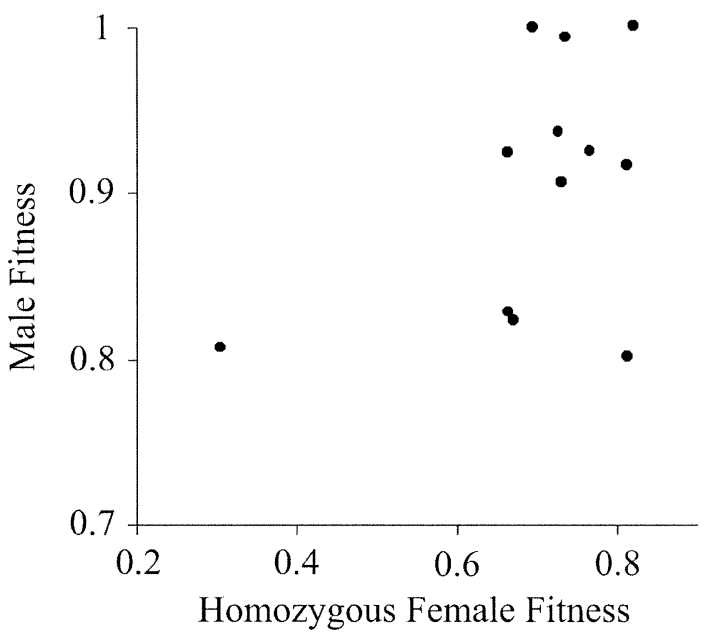

FIG. 4. The relationship between the fitness effects of experimental $\mathrm{X}$ chromosomes on (genotypically equivalent) males $\left(X_{i} / Y\right)$ versus homozygous females $\left(X_{i} X_{i}\right)$. The outlier $X_{5}$ is shown.

mons and Crow 1977; Crow 1993; Charlesworth and Charlesworth 1987, 1999). Mutation-selection balance theory predicts that purifying selection against recessive mutations occurs more readily for X-linked compared to autosomal loci, which should shift the frequency distribution of dominance coefficients upwards (as indicated by a study of X-linked egg-to-adult viability; Eanes et al. 1985). Under a pure mutation-selection balance model, the estimate of $h$ reported here would be surprising low. The sex-antagonistic model also predicts covariation between selection and dominance coefficients (Rice 1984), and hence, a positive $h$, although there is currently no empirical test of this prediction. The lack of correlation between homozygous and heterozygous females presented here supports the overdominance model. It should be noted that this particular line of evidence (though in agreement with the variance data; see above and Table 2) should be taken with caution as this test for dominance relies on large sample sizes.

\section{Fitness Correlation between Males and Females}

$\mathrm{X}$-chromosome fitness variation in males and homozygous females is uncorrelated $\left(X_{5}\right.$ removed: $R^{2}=0.028, P=0.26$; $X_{5}$ included: $R^{2}=0.244, P=0.10$ ) (Fig. 4). The slope of the regression line is not significantly different from zero, though confidence intervals are broad due to sample size $\left(X_{5}\right.$ removed: lower $95 \%=-0.501$, upper $95 \%=0.823 ; X_{5}$ included: lower $95 \%=-0.0865$, upper $95 \%=0.437)$.

The lack of correlation between the sexes could reflect mutational variation at sex-limited loci or sex-by-genotype interactions. Results from Table 2 differ from expectations of the sex-limited mutation-selection balance hypothesis (i.e., harmful mutations for females do not affect male fitness), which predicts that male fitness variance will be low relative to homozygous female variance. However, male fitness variation can be equal to or greater than homozygous female fitness variation if the mutation rate of male-limited genes is greater than that of female-limited genes. Because there is no a priori reason to expect such a discrepancy to arise (rather, the opposite might be expected since genes that are highly expressed in females, or "female-biased" genes, are much more numerous on the Drosophila X than are "male-biased", genes; Parisi et al. 2003; Ranz et al. 2003), this latter scenario is dubious.

The sexual antagonism model predicts a negative fitness correlation between the sexes, a pattern which we did not find. It is important to note, however, that if sexually antagonistic loci are particularly numerous on the $\mathrm{X}$, a large sample size is required in order to generate a negative intersexual fitness correlation because most $\mathrm{X}$ chromosomes will harbor a balance of female- and male-beneficial alleles, thereby concealing the range of naturally segregating variation (only a large sample will include "good male", and "good female", $\mathrm{X}$ chromosomes). The difference in relative levels of genetic variation between natural (i.e., from isofemale lines) and laboratory adapted Drosophila populations might explain why Gibson et al. (2002) demonstrated X-linked sexually antagonistic variation with a slightly larger sample $(n=20$ vs. $n$ $=12$ presented here).

\section{Future Directions}

Our study represents a creative approach to ascertain whether overdominant selection maintains much of the fitness variation that is commonly observed within animal populations. Future work will be required to better quantify the magnitude of overdominant fitness variation as well as the extent to which such conclusions can be extrapolated to describe autosomal variation.

It should be noted that the data support a model of overdominance, but such patterns can potentially stem from two genetic bases (see Charlesworth and Hughes 1999). Because we measure composite traits encompassing many individual characteristics, a pattern of overdominance can arise if heterozygosity is favored in one or more traits contributing to the fitness measurement ("pure", overdominance), or if fitness trade-offs exist between individual traits, causing higher heterozygote fitness when traits are summed (i.e., "net" overdominance, which includes antagonistic pleiotropy). Future research, involving many individual traits, will be required to discriminate between these possibilities.

Three sources of experimental noise can potentially limit the accuracy of these results. Theories discussed here assume that populations are at genetic equilibrium with respect to the environment in which fitness was tested, yet violation of this assumption is not expected to enhance patterns of overdominant selection. More precise estimates of the effect of overdominance can perhaps be accomplished by studying equilibrium, laboratory adapted populations, which differ from natural populations in some potentially profound ways (e.g., relatively constant population size over time; environmental stability), but are certainly adapted to the environment in which the experiment occurs (see Chippindale et al. 2001; Houle and Rowe 2003; Gardner et al. 2005).

Experimental populations might deviate from idealized populations (i.e., at $\mathrm{H}-\mathrm{W}$ and linkage equilibrium), although this is not likely to bias results towards patterns of overdominance. If overdominant selection is asymmetrical (i.e., $s \neq$ $t$; Table 1) and the standard $\mathrm{X}$ is enriched in rare alleles, most crosses will produce heterozygous genotypes. If en- 
riched in common alleles, most crosses will produce the mildly deleterious homozygous genotype. In either scenario, variance is expected to be reduced among the heterozygous class, limiting detection of overdominance (through the $V_{X_{i} X_{5}}: V_{X_{i} X_{i}}$ ratio). Linkage disequilibrium (LD) is also unlikely to account for results presented here, as it is predicted to have a negligible impact on measures of fitness variation (Charlesworth and Hughes 1999). Linkage disequilibrium is also expected to be quite low on the $\mathrm{X}$ because the effective recombination rate for Drosophila X-linked genes is high (Betancourt et al. 2004), and because X-linked inversion polymorphisms are extremely rare (Aulard et al. 2002).

Epistasis can arise from interactions between loci within experimental X chromosomes and other parts of the genome, and potentially introduces an additional source of fitness variation. Mutation-accumulation experiments indicate little to no epistasis between mutations (reviewed in Rice 2002), although instances of synergistic epistasis have occasionally been reported in Drosophila (Mukai 1969; Fry 2004; Rosa et al. 2005). Such epistatic variation will mainly be expressed in experimental homozygotes (Charlesworth and Hughes 1999) and will bias results away from overdominance predictions, making our results conservative.

Finally, the signature of overdominance is strongest earlier in the female fitness trials, a finding that might be due to mutations with late acting deleterious effects. Future studies that measure fitness variation expressed during the earliest adult period (i.e., less than four days posteclosion) are likely to detect the strongest signatures of overdominance.

\section{ACKNOWLEDGMENTS}

We would like to thank R. Huddleston, J. Kurdziel, P. Midford, E. Oneal, F. Paper, and R. Walters for help during the experiment, D. Bachtrog for providing the isofemale lines, and B. Carstens, M. Chatfield, E. Oneal, S. Otto, M. Noor, T. Wittkopp, and four anonymous reviewers for discussion and comments that greatly improved the manuscript. Research was funded by grants from the University of Michigan Rackham Graduate School and Sigma Xi to TC, and by a National Science Foundation grant (DEB-04-47224) to LLK.

\section{Literature Cited}

Aulard, S., J. R. David, and F. Lemeunier. 2002. Chromosomal inversion polymorphism in Afrotropical populations of Drosophila melanogaster. Genet. Res. 79:79-63.

Bateman, A. J. 1948. Intrasexual selection in Drosophila. Heredity $2: 349-368$.

Betancourt, A. J., Y. Kim, and H. A. Orr. 2004. A pseudohitchhiking model of X vs. autosomal diversity. Genetics 168:2261-2269.

Charlesworth, B., and D. Charlesworth. 1999. The genetic basis of inbreeding depression. Genet. Res. 74:329-340.

Charlesworth, B., and K. A. Hughes. 1999. The maintenance of genetic variation in life-history traits. Pp. 369-392 in R. S. Singh and C. B. Krimbas, eds. Evolutionary genetics: from molecules to morphology, Vol. 1. Cambridge Univ. Press, Cambridge, U.K.

Charlesworth, D., and B. Charlesworth. 1987. Inbreeding depression and its evolutionary consequences. Annu. Rev. Ecol. Syst. 18:237-268.

Chippindale, A. K., J. R. Gibson, and W. R. Rice. 2001. Negative genetic correlation for adult fitness between sexes reveals on- togenetic conflict in Drosophila. Proc. Natl. Acad. Sci. USA 98: $1671-1675$

Crow, J. F. 1993. Mutation, mean fitness, and genetic load. Pp. 342 , in D. Futuyma and J. Antovics, eds. Oxford surveys in evolutionary biology. Vol. 9. Oxford Univ. Press, New York.

Crozier, R. H. 1976. Why male-haploid and sex-linked genetic systems seem to have unusually sex-limited mutational genetic loads. Evolution 30:623-624.

Eanes, W. F., J. Hey, and D. Houle. 1985. Homozygous and hemizygous viability variation on the $\mathrm{X}$ chromosome of Drosophila melanogaster. Genetics 111:831-844.

Falconer, D. S., and T. F. C. Mackay. 1996. Introduction to quantitative genetics. 4th ed. Longman, Essex, U.K

Fisher, R. A. 1922. On the dominance ratio. Proc. R. Soc. Edinburgh $52: 312-341$

Fry, J. D. 2004. On the rate and linearity of viability declines in Drosophila mutation-accumulation experiments: genomic mutation rates and synergistic epistasis revisited. Genetics 166: 797-806.

Gardner, M. P, K. Fowler, N. H. Barton, and L. Partridge. 2005. Genetic variation for total fitness in Drosophila melanogaster: complex yet replicable patterns. Genetics 169:1553-1571.

Gibson, J. R., A. K. Chippindale, and W. R. Rice. 2002. The X chromosome is a hot spot for sexually antagonistic fitness variation. Proc. R. Soc. Lond. B 269:499-505.

Greenberg, R., and J. F. Crow. 1960. A comparison of the effect of lethal and detrimental chromosomes from Drosophila populations. Genetics 45:1153-1163.

Hartl, D. L., and A. G. Clark. 1997. Principles of population genetics. 3rd ed. Sinauer, Sunderland, MA.

Hedrick, P. W. 1985. Genetics of populations. Jones and Bartlett, Boston, MA.

Hedrick, P. W., and J. D. Parker. 1997. Evolutionary genetics and genetic variation of haplodiploids and X-linked genes. Annu. Rev. Ecol. Syst. 28:55-83.

Holland, B. and W. R. Rice. 1999. Experimental removal of sexual selection reverses intersexual antagonistic coevolution and removes a reproductive load. Proc. Natl. Acad. Sci. USA 96: 5083-5088.

Houle, D., and L. Rowe. 2003. Natural selection in a bottle. Am. Nat. 161:50-67.

Hughes, K. A., and B. Charlesworth. 1994. A genetic analysis of senescence in Drosophila. Nature 367:64-66.

James, J. W. 1973. Covariances between relatives due to sex-linked genes. Biometrics 29:584-588.

Lewontin, R. C. 1974. The genetic basis of evolutionary change. Columbia Univ. Press, New York.

Medawar, P. B. 1952. An unsolved problem of biology. Lewis, London.

Mukai, T. 1969. Genetic structure of natural populations of Drosophila melanogaster. 7. Synergistic interaction of spontaneous mutant polygenes controlling viability. Genetics 61:749-1969.

Mukai, T., and O. Yamaguchi. 1974. The genetic structure of natural populations of Drosophila melanogaster. XI. Genetic variability in a local population. Genetics 76:339-366.

O'Brien, R. G. 1981. A simple test for variance effects in experimental designs. Psych. Bull. 89:570-574.

Pamilo, P. 1979. Genic variation at sex-linked loci: quantification of regular selection models. Hereditas 91:129-133.

Parisi, M., R. Nuttall, D. Naiman, G. Bouffard, J. Malley, J. Andrews, S. Eastman, and B. Oliver. 2003. Paucity of genes on the Drosophila X chromosome showing male-biased expression. Science 299:697-700.

Partridge, L. 1980. Mate choice increases a component of offspring fitness in fruit flies. Nature 283:290-291.

Promislow, D. E. L., M. Tatar, A. Khazaeli, and J. W. Curtsinger. 1996. Age-specific patterns of genetic variance in Drosophila melanogaster. I. Mortality. Genetics 143:839-848.

Ranz, J. M., C. I. Castillo-Davis, C. D. Meiklejohn, and D. L. Hartl 2003. Sex-dependent gene expression and evolution of the Drosophila transcriptome. Science 300:1742-1745.

Rice, W. R. 1984. Sex chromosomes and the evolution of sexual dimorphism. Evolution 38:735-742. 
Rice, W. R. 2002. Experimental tests of the adaptive significance of sexual recombination. Nat. Rev. Genet. 3:241-251.

Rosa, J. M., S. Camacho, and A. Garcia-Dorado. 2005. A measure of the within-chromosome epistasis for Drosophila viability. J. Evol. Biol. 18:1130-1137.

Saccheri, I. J., H. D. Lloyd, S. J. Helyar, and P. M. Brakefield. 2005. Inbreeding uncovers fundamental differences in the genetic load affecting male and female fertility in a butterfly. Proc. R. Soc. Lond. B 272:39-46.

Simmons, M. J., and J. F. Crow. 1977. Mutations affecting fitness in Drosophila populations. Ann. Rev. Genet. 11:49-78.

Sokal, R. R., and F. J. Rohlf. 1995. Biometry. 3rd ed. W. H. Freeman, New York.

Tracey, M. L., and F. J. Ayala. 1974. Genetic load in natural pop- ulations: is it compatible with the hypothesis that many polymorphisms are maintained by natural selection? Genetics 77: 569-589.

Trivers, R. L. 1972. Parental investment and reproductive success. Pp. 136-179 in B. Campbel, ed. Sexual selection and the descent of man. Aldine-Atherton, Chicago.

Wilton, A. D., and J. A. Sved. 1979. X chromosomal heterosis in Drosophila melanogaster. Genet. Res. 34:303-315.

Zeh, J. A., and D. W. Zeh. 1996. The evolution of polyandry. I. Intragenomic conflict and genetic incompatibility. Proc. R. Soc. Lond. B 263:1711-1717.

Zhang, X.-S., and W. G. Hill. 2005. Genetic variability under mutation selection balance. Trends Ecol. Evol. 20:468-470.

Corresponding Editor: M. Noor 